\title{
IgA is important for clearance and critical for protection from rotavirus infection
}

\author{
SE Blutt ${ }^{1}$, AD Miller $^{1}$, SL Salmon ${ }^{2}$, DW Metzger ${ }^{2}$ and ME Conner ${ }^{1}$
}

\begin{abstract}
Based on a lack of severe phenotype in human immunoglobulin $A(\lg A)$ deficiency syndromes, the role of $\lg A$ in controlling respiratory and gastrointestinal (GI) infections has not been clearly defined. C57BL/6 and BALB/c mice lacking $\left.\lg \mathrm{A}_{\left(\lg \mathrm{A}^{-/-}\right.}\right)$were developed and used to address this question. When exposed to a common GI virus, rotavirus, $\lg A^{-/-}$mice exhibited a substantial and significant delay in clearance of the initial infection compared with wild-type mice. IgA-/- mice excreted rotavirus in stool up to 3 weeks after the initial exposure compared with 10 days observed in wild-type mice. Importantly, IgA $\mathrm{A}^{-/-}$mice failed to develop protective immunity against multiple repeat exposures to the virus. All IgA-/- mice excreted virus in the stool upon re-exposure to rotavirus, whereas wild-type mice were completely protected against re-infection. These findings clearly indicate a critical role for IgA in the establishment of immunity against a Gl viral pathogen.
\end{abstract}

\section{INTRODUCTION}

Immunoglobulin A (IgA) is the most abundant antibody isotype found in the body ${ }^{1}$ and has an important role in the immune responses at mucosal surfaces such as the gastrointestinal (GI) tract, the respiratory tract, and the vaginal tract. ${ }^{2}$ It mediates its effector function through multiple mechanisms, ${ }^{3}$ including interactions with mucosal epithelial cells, binding to a receptor, and high- and low-affinity antigen binding. IgA clearly has a multi-faceted role in mucosal immunity but whether IgA alone is critical to immunity against pathogens that invade and cause disease at mucosal surfaces remains unresolved. Although some humans lacking IgA suffer from recurrent sinopulmonary and/ or GI infections, ${ }^{4}$ many exhibit no apparent phenotype, ${ }^{4}$ suggesting that $\operatorname{IgA}$ is not absolutely essential to combat mucosal pathogens.

To attempt to determine the significance of IgA production at the mucosal surface, IgA knockout mice were generated by deleting the IgA switch region and half of the constant region. ${ }^{5}$ These mice are of mixed $129 \times \mathrm{C} 57 \mathrm{Bl} / 6$ background (C57BL/6/129 $\mathrm{IgA}^{-/-}$) and lack IgA antibody-secreting cells and thus have no detectable IgA in either serum or mucosal secretions. ${ }^{5}$ Presumably to compensate for the loss of IgA, the $\mathrm{C} 57 \mathrm{BL} / 6 / 129 \mathrm{IgA}^{-/-}$mice have elevated levels of IgM and IgG compared with wild-type mice. ${ }^{5}$ There have been limited studies examining whether the absence of IgA results in diminished immune responses to mucosal pathogens.
Consistent with the evidence in humans that the lack of IgA does not have a devastating impact on mucosal immunity, infection of C57BL/6/129 IgA-/- mice with Herpes simplex virus, Helicobacter pylori, or influenza virus showed that IgA is not essential for clearance of or protection from these mucosal pathogens. ${ }^{6-8}$ However, IgA was essential to block non-enteropathogenic reovirus entry in Peyer's patches. ${ }^{9}$ Presumably, sites of infection and the pathogenesis of individual pathogens are expected to greatly influence the relative importance of IgA in protection.

Rotavirus is a common intestinal virus that causes acute diarrhea in humans and animals. ${ }^{10}$ Small intestinal absorptive epithelial cells are the primary site of virus replication and pathology. ${ }^{10}$ In humans and animals, intestinal rotavirus-specific IgA is a correlate of protection from rotavirus ${ }^{10}$ suggesting that intestinal IgA may be essential in the immune response to rotavirus. To directly examine this possibility, we previously tested the outcome of rotavirus infection in $\mathrm{C} 57 \mathrm{BL} / 6 / 129 \mathrm{IgA}^{-1-}$ mice. We found that IgA was essentially dispensable for clearance of an initial rotavirus infection ${ }^{11,12}$ and for protection from re-infection, ${ }^{11}$ the latter attributed to compensatory responses in IgM and IgG antibodies. ${ }^{11}$ This report, at the time, was consistent with the limited role of $\operatorname{IgA}$ in immune responses to influenza, Herpes simplex virus, and $H$. pylori. ${ }^{6-8}$

Since our initial report, several other reports examined the role of either B cells or components of the IgA secretory

${ }^{1}$ Department of Molecular Virology and Microbiology, Baylor College of Medicine, Houston, Texas, USA. ${ }^{2}$ Center for Immunology \& Microbial Disease, Albany Medical College, Albany, New York, USA. Correspondence: ME Conner (mconner@bcm.tmc.edu)

Received 20 September 2011; accepted 1 May 2012; published online 27 June 2012. doi:10.1038/mi.2012.51 
pathway in rotavirus immunity and the conclusions from these studies are seemingly inconsistent with our initial conclusion that IgA is dispensable for rotavirus immunity. Mice lacking $\mathrm{B}$ cells (either JhD or $\mu \mathrm{Mt}$ mice on $\mathrm{C} 57 \mathrm{Bl} / 6$ background) are not protected from rotavirus re-infection, ${ }^{13,14}$ clearly suggesting antibody is an important player in the intestinal immune response to rotavirus. Similarly, we found that the JhD mice on a $B A L B / c$ background have a greatly impaired ability to clear a primary rotavirus infection, ${ }^{15}$ supporting a role for $\mathrm{B}$ cells in clearance of infection. The requirement for $\mathrm{B}$ cells does not directly prove that antibody or IgA is a critical factor, but IgA is certainly the predominant Ig produced in the intestine during rotavirus infection. ${ }^{10}$ Both rotavirus-specific $\operatorname{Ig} \mathrm{A}$ and $\operatorname{IgG}$ have been implicated in protective immunity. ${ }^{12,16}$ Additional support for the importance of IgA to rotavirus immunity comes from studies in J-chain-deficient mice, which cannot transcytose IgA or IgM into the intestinal lumen. J chain ${ }^{-1-}$ mice have difficulty clearing a primary rotavirus infection ${ }^{17}$ and are not protected from re-infection. ${ }^{17,18}$ Therefore, rotavirus infection in mice lacking either B cells or secretory antibodies (IgA and IgM) at mucosal surfaces results in similar defects in clearance of rotavirus infection and protection from re-infection. However, because IgM is also affected by the loss of B cells or J chain, these studies fail to definitively prove that IgA is critical for rotavirus immunity. ${ }^{19}$ As more information has been gathered on the important cells and effector molecules required for rotavirus immunity, we felt it was necessary to reexamine the conclusion that IgA is not required for immunity to rotavirus.

Genetic background can dramatically influence the susceptibility to rotavirus infection in the mouse model with $\mathrm{BALB} / \mathrm{c}$ mice being $1,000 \times$ more susceptible than C57BL/ 6 mice to the murine rotavirus strain $\mathrm{EC}_{\mathrm{wt}}{ }^{20}$ As the phenotype of a mutation can be influenced by genetic background ${ }^{21}$ and our previous data was collected in mice of a mixed genetic background, ${ }^{11}$ we backcrossed mice with the IgA mutation onto C57BL/6 and $\mathrm{BALB} / \mathrm{c}$ genetic backgrounds $\left(\mathrm{C} 57 \mathrm{BL} / 6 \mathrm{IgA}^{-1-}\right.$ and $\mathrm{BALB} / \mathrm{c}$ $\left.\mathrm{IgA}^{-/-}\right)$and reassessed the affects of clearance of rotavirus infection and protection from re-infection in each strain. Irrespective of genetic background, $\mathrm{IgA}^{-1-}$ mice exhibited major defects in clearance of the initial rotavirus infection and the development of protective immunity. These results clearly demonstrate the importance of intestinal IgA to timely clearance of an initial infection with rotavirus and the absolute necessity for IgA in the establishment of protective immunity against re-infection in the murine model of rotavirus.

\section{RESULTS}

\section{Clearance of rotavirus infection in mice coincides with the induction of fecal IgA}

IgA has long been implicated as an important factor in protective immunity against rotavirus in humans and animal models of rotavirus infection and disease. ${ }^{10}$ To demonstrate that mice on two different genetic backgrounds exhibit a similar induction of intestinal IgA following exposure to a $10^{3} \mathrm{ID}_{50}$ dose ( $50 \%$ of the infectious dose) of murine rotavirus, $\mathrm{C} 57 \mathrm{BL} / 6$ and
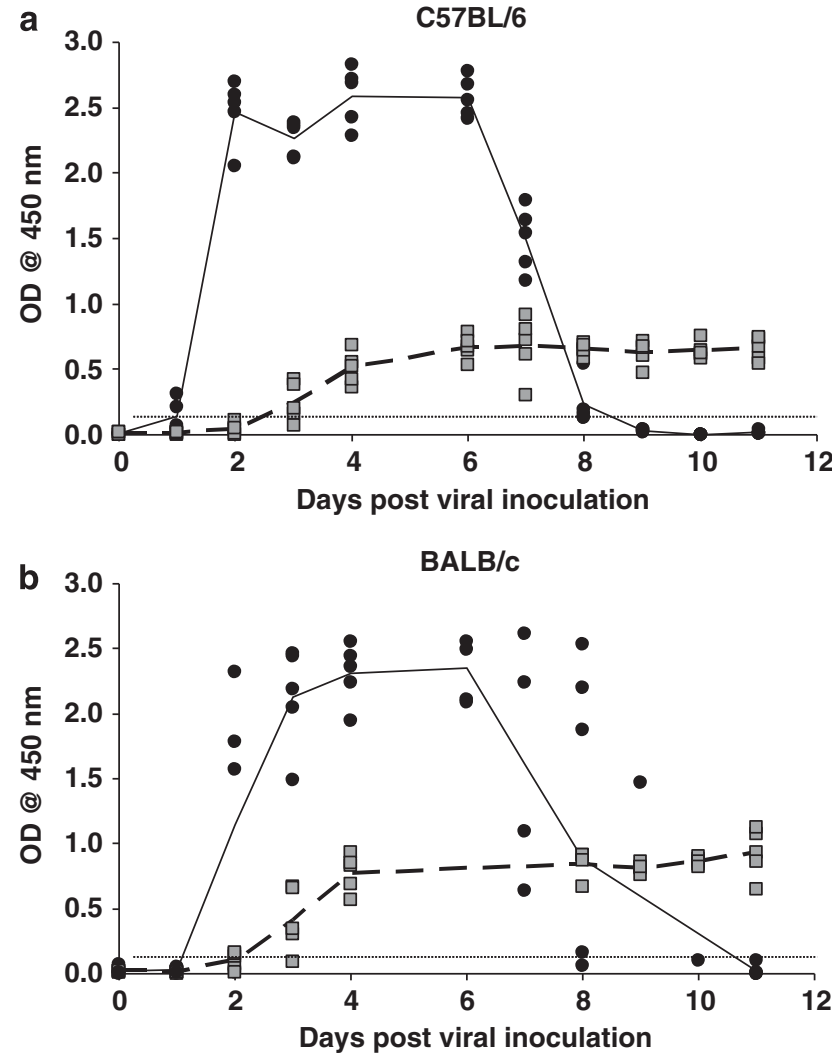

Figure 1 Induction of fecal immunoglobulin A ( $\lg A)$ coincides with clearance of rotavirus infection in mice. (a) C57BL/6 and (b) BALB/c mice were orally inoculated with $10^{3} I_{50}\left(50 \%\right.$ of the infectious dose) of $E C_{w t}$ rotavirus on day 0 . Fecal pellets were collected every day and analyzed for the presence of the VP6 middle capsid protein by enzyme-linked immunosorbent assay (mean values shown by solid line) or the presence of rotavirus-specific IgA (mean value shown by dotted line). Each symbol represents the optical density (OD) of either antigen (black circles) or antibody (gray squares) present in the stool of an individual mouse. Horizontal line indicates the limit of detection of the assay.

$\mathrm{BALB} / \mathrm{c}$ mice were orally inoculated with rotavirus and stool assessed for the presence of rotavirus antigens or rotavirus-specific IgA by the enzyme-linked immunosorbent assay (ELISA). As expected, rotavirus was detected in stool of mice from both the genetic backgrounds by 2 days following virus exposure and was no longer detectable by 10 days (Figure 1). Rotavirus-specific IgA in stool was detected in both strains as early as 3 days following viral inoculation (Figure 1). As previously reported, ${ }^{22}$ the appearance of rotavirus-specific IgA in the stool preceded the disappearance of rotavirus from the fecal isolates. These findings indicate that induction of rotavirus-specific $\operatorname{IgA}$ is similar on both the genetic backgrounds and suggest that intestinal IgA is an important factor in the resolution of rotavirus infection.

\section{Clearance of rotavirus infection in mice lacking IgA on a homologous genetic background is significantly delayed}

To re-examine whether IgA was important for clearance of a rotavirus infection, $\mathrm{C} 57 \mathrm{BL} / 6$ and $\mathrm{BALB} / \mathrm{c} \mathrm{IgA}^{-/-}$and wild-type 
mice were orally inoculated with $\mathrm{EC}_{\mathrm{wt}}$. The kinetics of virus shedding between wild-type and IgA ${ }^{-/-}$strains were initially similar at onset of shedding and in the subsequent sharp decline in shedding that occurred between 6-8 days following virus inoculation (Figure 2a,b). Although all wild-type mice of both backgrounds resolved infection by 10 days, all $\operatorname{IgA}^{-1-}$ mice continued to shed virus through 20-22 days following inoculation albeit at reduced levels compared with peak shedding (Figure 2a,b). There was a significant difference in the mean number of time points that rotavirus was detectable in the stool (Figure 2c) between C57BL/6 $\mathrm{IgA}^{-1-}$ and wild-type mice (6.4 vs. 3.8 time points; $P=0.003)$ and $\mathrm{BALB} / \mathrm{c} \mathrm{IgA}^{-/-}$and wild-type mice (6.5 vs. 3.2 time points; $P=0.000$ ). The endpoint titer of virus excreted in stool at the peak of viral shedding (day 4 for $\mathrm{BALB} / \mathrm{c}$ strains and day 6 for $\mathrm{C} 57 \mathrm{BL} / 6$ strains) was determined by limiting dilution. The geometric mean antigen titer was 2.6-3-fold higher in the IgA ${ }^{-1-}$ mice but was not significantly different from the respective wild-type controls (Table 1). The $\mathrm{IgA}^{-1-}$ mice did not appear to have enhanced susceptibility to rotavirus infection compared with wild-type mice (data not shown); however, loss of IgA clearly affected the ability of mice to resolve rotavirus infection.

\section{Mice lacking IgA are not protected from re-infection with rotavirus}

To determine whether the deletion of IgA also altered protection from re-infection, C57BL/ 6 and $\mathrm{BALB} / \mathrm{c} \mathrm{IgA}^{-/-}$and wild-type mice previously infected with rotavirus were re-inoculated with rotavirus 6 weeks later. Susceptibility to re-infection was assessed based on viral excretion in stool. All C57BL/6 and BALB/c $\mathrm{IgA}^{-/-}$mice were susceptible to re-infection and excreted substantial levels of rotavirus in the stool for multiple days (Figure 3a,b). By contrast, all the wild-type mice were completely resistant to re-infection. The mean number of time points virus that was present in the stool (Figure 3c) was significantly higher in the knockout compared with the wild-type mice (C57BL/6: 4.8 vs. $0, P=0.004 ; \mathrm{BALB} / \mathrm{c}: 4.8$ vs. $0, P=0.005$ ). The C57BL/6 IgA I- $^{-1}$ mice exhibited an eightfold higher geometric mean antigen at peak day of viral excretion (day 4 for both genetic strains) compared with $\mathrm{BALB} / \mathrm{c} \mathrm{IgA}^{-/-}$mice (Table 1) but the difference was not significant.

To examine whether establishment of protective immunity against rotavirus exposure was merely delayed in the absence of IgA production, $\mathrm{C} 57 \mathrm{BL} / 6$ or $\mathrm{BALB} / \mathrm{c} \operatorname{IgA}{ }^{-/-}$and wild-type mice were challenged with rotavirus at 12 , instead of 6 , weeks following the initial viral exposure. As expected, ${ }^{22}$ all wild-type mice were protected against re-infection and did not excrete any detectable levels of rotavirus in stool. However, all $\operatorname{IgA}^{-1-}$ mice excreted rotavirus in the stool (Figure 3a,b) and for a significantly greater number of time points (Figure 3c) compared with wild-type mice (C57BL/6: 3.2 vs. $0, P=0.004 ; \mathrm{BALB} / \mathrm{c}: 1.7$ vs. 0 , $P=0.003)$. The C57BL/6 IgA ${ }^{-1-}$ mice had a 17.6-fold higher geometric mean titer of viral antigen on the day of peak viral shedding following challenge compared with $\mathrm{BALB} / \mathrm{c} \operatorname{Ig} \mathrm{A}^{-/-}$ mice (day 4 for both strains) but the difference was not significance (Table 1). There was no difference in the geometric
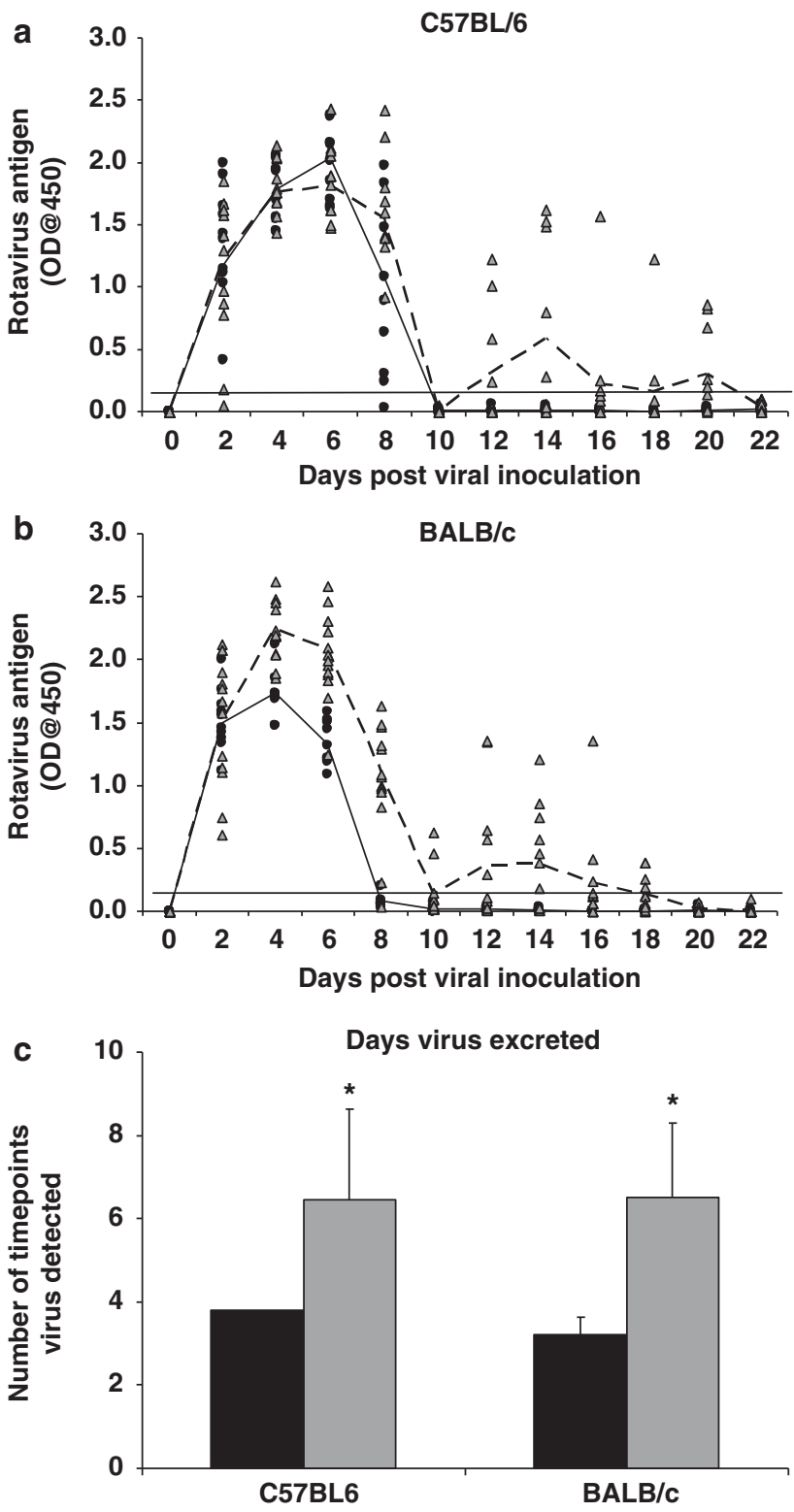

Figure 2 Immunoglobulin A $(\lg A)$ is important for clearance of rotavirus infection. (a) C57BL/6 and (b) BALB/c mice were orally inoculated with $10^{3} \mathrm{ID}_{50}\left(50 \%\right.$ of the infectious dose) of $\mathrm{EC}_{\mathrm{wt}}$ rotavirus on day 0 . Fecal pellets were collected every other day and analyzed for the presence of the VP6 middle capsid protein by enzyme-linked immunosorbent assay. Each symbol represents the optical density (OD) of VP6 present in an individual wild-type (black circles) or $\lg \mathrm{A}^{-/-}$(gray triangles) mouse. The mean OD values for the groups are indicated for wild-type (solid line) and $\lg \mathrm{A}^{-1-}$ (dotted line) mice. Horizontal line indicates the limit of detection of the assay. (c) The mean number of time points antigen was detected in fecal samples for each group. Black bar, wild type; grey bar, $\lg \mathrm{A}^{-/-}$. Each bar represents the mean for each group + s.d. ( $n=10-12$ mice/group). ${ }^{\star} P<0.05$ by Kruskal-Wallis equality of populations rank test compared with wild-type mice.

mean titer of virus excreted at 6 weeks compared with 12 weeks in either genetic background (Table 1). These results indicate that $\operatorname{IgA}$ is critical for the establishment of protective immunity against rotavirus infection. 
Table 1 Geometric mean titer (range) of rotavirus antigen in $10 \%$ (wt/v) stool samples on peak day of virus shedding at indicated inoculation ${ }^{1}$

\begin{tabular}{|c|c|c|c|c|}
\hline Mouse strain $(n=5)$ & Primary & \multicolumn{2}{|c|}{ Secondary } & Tertiary \\
\hline C57BL/6 & $460(200-1,600)$ & 0 & 0 & 0 \\
\hline $\mathrm{BALB} / \mathrm{c}$ & $696(200-1,600)$ & 0 & 0 & 0 \\
\hline $\mathrm{BALB} / \mathrm{c} \lg \mathrm{A}^{-1-}$ & $2,111(800-25,600)$ & $17(5-2,560)$ & $6(5-10)$ & $6(5-10)$ \\
\hline
\end{tabular}

Abbreviations: IgA, immunoglobulin A; wt/v, weight/volume.

${ }^{1}$ At all inoculations, mice were administered $10^{3} \mathrm{ID}_{50} \mathrm{EC}_{\mathrm{wt}}\left(50 \%\right.$ of the infectious dose of the murine strain of rotavirus $\left.\mathrm{EC}_{\mathrm{wt}}\right)$.

\section{IgA is essential for protection against repeated rotavirus infections}

To determine whether IgA $\mathrm{A}^{-1-}$ mice were susceptible to repeated infections, $\mathrm{C} 57 \mathrm{BL} / 6$ and $\mathrm{BALB} / \mathrm{CIgA}^{-1-}$ and wild-type mice were inoculated with rotavirus three times ( 0,6 , and 12 weeks). Both C57BL/6 and BALB/c IgA ${ }^{-/-}$mice remained susceptible to repeated infection (Figure 4a,b) unlike wild-type mice that were fully protected following initial infection (data not shown). There was no difference in the geometric mean titer of virus excreted at peak viral excretion (4 days) between the first and the second challenge (Table 1). However, rotavirus was excreted for significantly fewer time points (Figure 4c) by all IgA $\mathrm{A}^{-/-}$mice following the third infection (12 weeks) compared with the second infection (6 weeks) (C57BL/6: 4.8 vs. 2.4, $P=0.006$; BALB/c: 2.4 vs. $1.0, P=0.015$ ). There was no difference in the mean number of time points positive for rotavirus at 12 weeks independent of a second inoculation at 6 weeks (C57BL/6: 2.4 vs. $3.2, P=0.340$; BALB/c: 1.0 vs. $1.7, P=0.162$ ). There was also no difference in geometric mean titer of virus excreted at peak viral infection (4 days) between the first and the second challenge (Table 1 ). In contrast to wild-type mice, none of the $\mathrm{C} 57 \mathrm{BL} / 6$ or the $\mathrm{BALB} / \mathrm{c} \mathrm{IgA}^{-/-}$mice established complete protective immunity to rotavirus infection even after multiple virus exposures.

\section{IgA has a minimal role in clearance of rotavirus from the systemic circulation}

Rotavirus infection is not limited to the intestine and also involves viremia and antigenemia. ${ }^{23}$ To determine whether the lack of IgA had any effect on rotavirus antigenemia, C57BL/6 and $\mathrm{BALB} / \mathrm{c}$ IgA- $\mathrm{A}^{-1}$ or wild-type mice were orally administered rotavirus, and antigenemia assessed at the peak of viral excretion in stool (4 days) or when virus is no longer detectable in wild-type animals but still present in the $\operatorname{Ig} \mathrm{A}^{-/-}$animals (15 days). Both the wild-type mice and $\operatorname{Ig} \mathrm{A}^{-/-}$mice had high and equivalent levels of rotavirus antigenemia 4 days following initial infection (Figure 5). There were small but significant differences between wild-type and $\operatorname{IgA}^{-1-}$ mice in the levels of antigenemia at 15 days post inoculation, but the levels were dramatically reduced compared with the levels present at 4 days following infection (Figure 5). In contrast to what was observed in stool, virus was not detected in the blood of any wild-type or IgA $\mathrm{A}^{-/-}$mice following a second (6 week) or third (12 week) rotavirus inoculation (data not shown). These results suggest that IgA has a minor role in the clearance of a primary rotavirus infection from systemic circulation and is not required for protection against rotavirus antigenemia following re-exposure to the virus. In addition, the high levels of rotavirus-specific serum antibody induced in all the infected $\operatorname{Ig} \mathrm{A}^{-/-}$mice following primary infection (data not shown) did not protect these mice from rotavirus re-infection of the intestine.

\section{Mice lacking IgA do not develop detectable compensatory rotavirus-specific antibody responses following rotavirus infection}

To examine whether rotavirus-specific intestinal IgG or IgM is induced in $\operatorname{IgA}^{-1-}$ mice, $\mathrm{C} 57 \mathrm{BL} / 6$ and $\mathrm{BALB} / \mathrm{c} \mathrm{IgA} \mathrm{A}^{-/-}$and wild-type mice were inoculated with rotavirus, and 15 days later, total rotavirus-specific (IgA, IgG, and IgM) in stool was quantified by ELISA. ${ }^{24}$ None of the C57BL/ 6 or the BALB/C $\mathrm{IgA}^{-1-}$ mice had detectable rotavirus-specific total antibody (Figure 6). Similar results were obtained in stool at 6 and 12 weeks following infection with the virus (data not shown). These findings indicate that, in addition to not producing rotavirusspecific IgA, these mice also failed to produce detectable levels of rotavirus-specific IgM or IgG.

\section{DISCUSSION}

We find that loss of IgA seriously impacted rotavirus immunity. Clearance of a primary rotavirus infection was significantly and similarly delayed in $\mathrm{C} 57 \mathrm{BL} / 6$ and $\mathrm{BALB} / \mathrm{c} \mathrm{IgA}^{-1-}$ mice compared with wild-type mice. Although wild-type mice previously infected with rotavirus were completely protected from infection, none of the IgA $\mathrm{A}^{-/-}$mice were protected from a second infection either 6 or 12 weeks following the initial infection. Complete protective immunity was not induced in $\operatorname{IgA}^{-/-}$mice by repeated exposure to rotavirus infection. These results clearly demonstrate the critical importance of IgA in rotavirus intestinal immunity.

Our data also provide the first direct experimental evidence that pathogen-specific IgA is essential for the development of protective intestinal immunity to an enteropathogen. Indisputable in vivo experimental proof under normal physiological conditions that IgA alone is critical to immunity against pathogens, which invade and cause disease at any mucosal surfaces, has been lacking. In fact, IgA has only previously been 

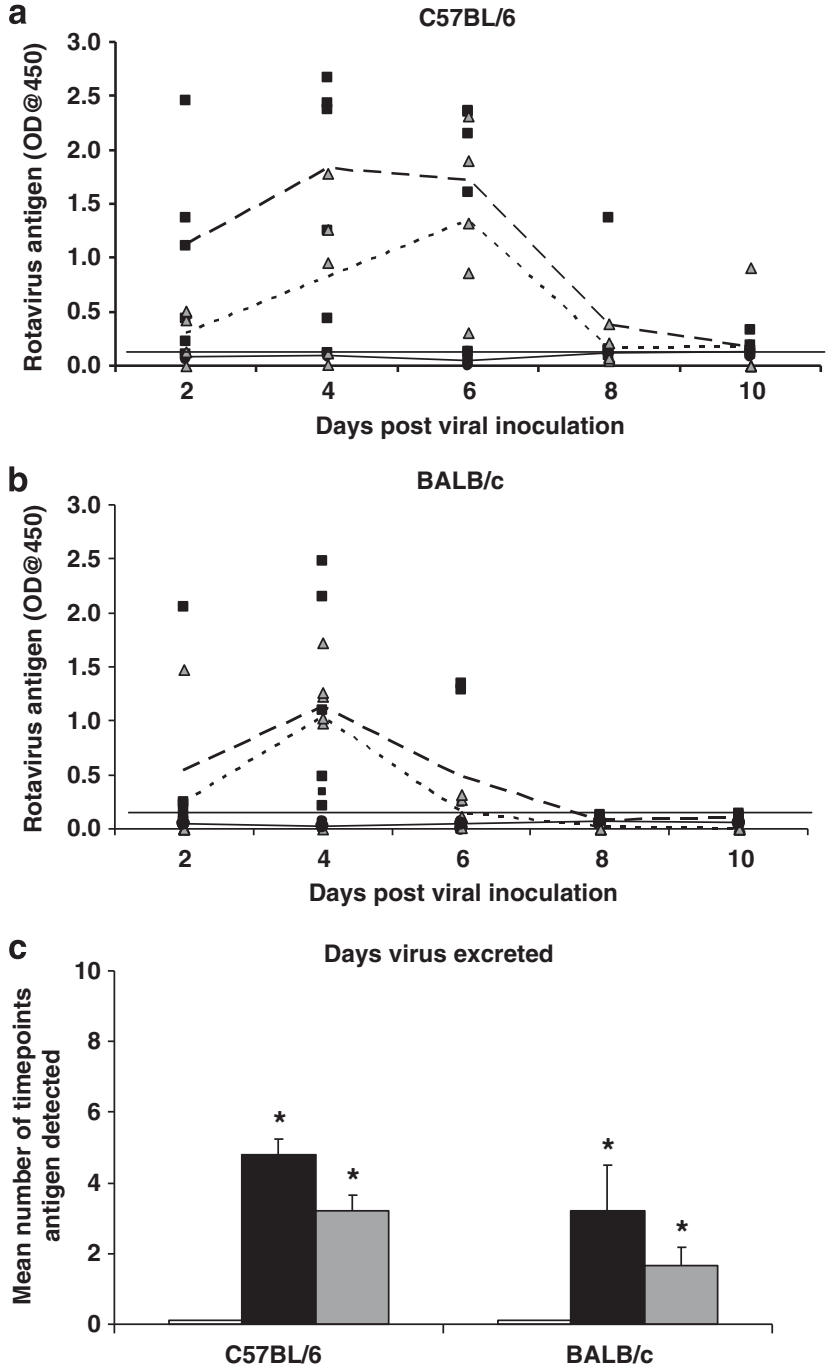

Figure 3 Immunoglobulin $A(\lg A)$ is required for protection from a secondary rotavirus infection. (a) C57BL/6 and (b) BALB/c wild-type or $\lg A^{-/-}$mice were orally inoculated with $10^{3} I_{50}$ (50\% of the infectious dose) of $E_{\mathrm{wt}}$ rotavirus and received a secondary inoculation with $10^{3}$ $\mathrm{ID}_{50} \mathrm{EC}_{\mathrm{wt}}$ either 6 or 12 weeks later. Fecal pellets were collected every other day and analyzed for the presence of the VP6 middle capsid protein by enzyme-linked immunosorbent assay. Each symbol represents the optical density (OD) obtained from an individual wild-type mouse (black circles) or IgA ${ }^{-/-}$mouse at 6 weeks (black squares) or 12 weeks (gray triangles). The mean antigen OD values for the groups are indicated for wild type (solid line) and $\lg \mathrm{A}^{-/-}$at either 6 weeks (large dotted line) or 12 weeks (small dotted line). Horizontal line indicates the limit of detection of the assay. (c) The mean number of time points antigen was detected in fecal samples for each group. White bar, wild-type mice, 6 or 12 week challenge (no antigen shedding at either time point); black bar, IgA ${ }^{-/-}$mice, 6-week challenge; gray bar, $\lg A^{-/-}$mice, 12-week challenge. Each bar represents the mean for each group +s.d. $(n=5-6$ mice/group). ${ }^{*} P<0.05$ by Kruskal-Wallis equality of populations rank test compared with wild-type mice.

shown to be critical to prevent non-enteropathogenic reovirus invasion of small intestinal Peyer's patches but is otherwise dispensable to reovirus immunity. ${ }^{9}$ Some studies with enteric bacterial pathogens or toxins or viruses in mice deficient in secretory antibodies ( $\operatorname{IgM}$ and $\operatorname{Ig} \mathrm{A}$ ) indicate that these antibodies are not
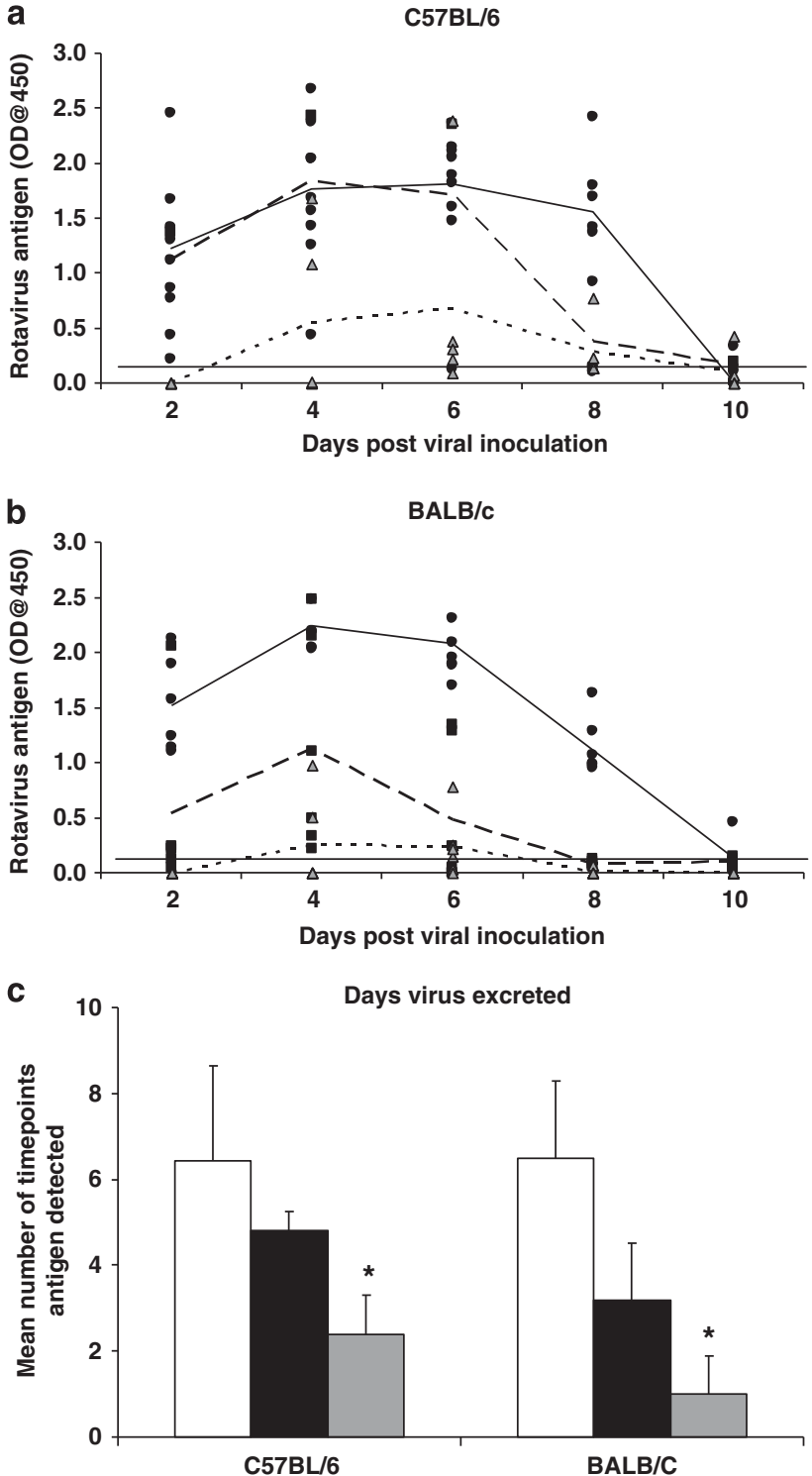

Figure 4 Mice lacking immunoglobulin A ( $\lg A)$ are susceptible to multiple infections with rotavirus. Naive (a) $\mathrm{C} 57 \mathrm{BL} / 6 \lg \mathrm{A}^{-/-}$or (b) BALB/C $\lg A^{-1-}$ mice were orally inoculated with $10^{3} I D_{50}(50 \%$ of the infectious dose) of $E_{\mathrm{wt}}$ rotavirus (mean value, solid line) followed by an identical second inoculation 6 weeks (mean value, large dotted line) and third inoculation 12 weeks later (mean value, small dotted line). Fecal pellets were collected every other day and analyzed for the presence of the VP6 middle capsid protein by enzyme-linked immunosorbent assay. Each symbol is the optical density (OD), representative of the amount of VP6 present in a sample from an individual IgA ${ }^{-/-}$mouse following the first (black circles), second (black squares, 6 weeks), and third (gray triangles, 12 weeks) inoculations. The mean antigen $\mathrm{OD}$ values for the groups are indicated following first (solid line), second (large dotted line), or third (small dotted line) inoculation. Horizontal line indicates the limit of detection of the assay. (c) The mean number of time points antigen was detected in fecal samples for each group. White bars, initial infection; black bars, 6-week inoculation; grey bars, 12-week inoculation. Each bar represents the mean for each group $+s . d$. ( $n=5-6$ mice per group). ${ }^{\star} P<0.05$ by Kruskal-Wallis equality of populations rank test compared mice receiving a challenge at 6 weeks. 


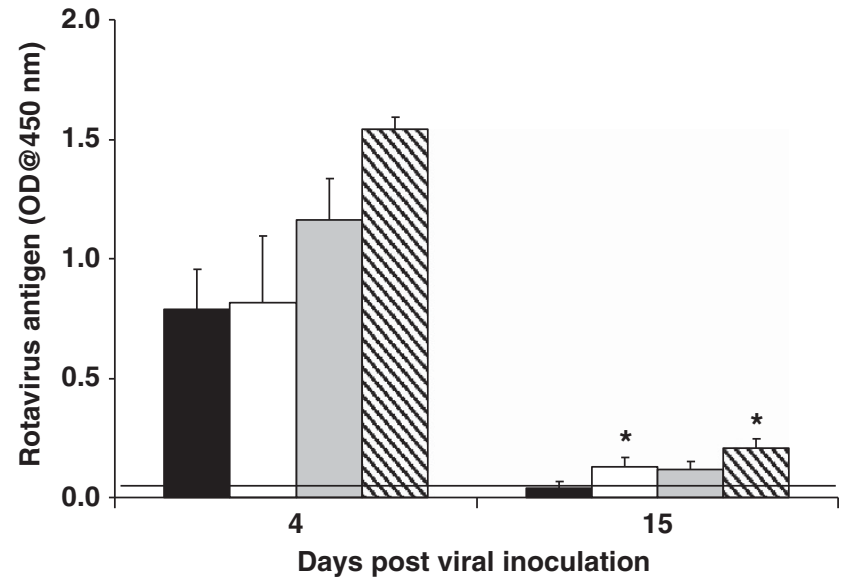

Figure 5 Immunoglobulin A $(\lg A)$ is not critical for clearance of rotavirus antigenemia. Mice were orally inoculated with $10^{3} I_{50}(50 \%$ of the infectious dose) of $\mathrm{EC}_{\mathrm{wt}}$ rotavirus on day 0 . Serum was collected either 4 or 15 days later and analyzed for the presence of the VP6 middle capsid protein by enzyme-linked immunosorbent assay. C57BL/6 (black bar), C57BL/6 IgA ${ }^{-/-}$(white bar), BALB/c (gray bar), and BALB/c $\operatorname{Ig} A^{-1-}$ (hatched bar). Each bar represents the mean optical density (OD) of VP6 present in 5-6 mice + s.d. ${ }^{*} P<0.05$ by Kruskal-Wallis equality of populations rank test compared with wild-type mice.

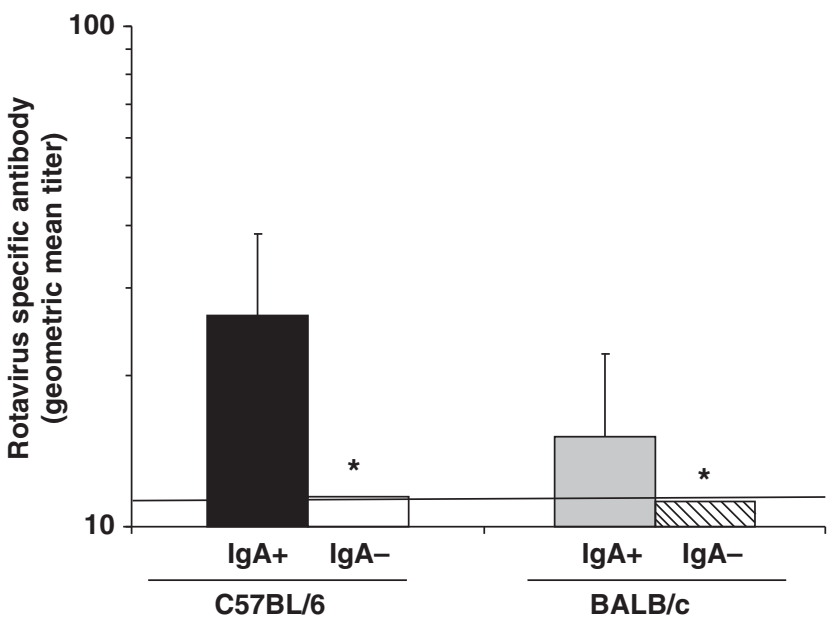

Figure 6 Mice lacking immunoglobulin A $(\lg A)$ do not develop detectable compensatory rotavirus-specific IgG or IgM fecal antibody. Mice were orally inoculated with $10^{3} I D_{50}$ (50\% of the infectious dose) of $\mathrm{EC}_{\mathrm{wt}}$ rotavirus on day 0 . Fecal pellets were analyzed for the presence of rotavirus-specific total antibody (IgA, IgM, and IgG) 15 days following viral exposure by enzyme-linked immunosorbent assay. Each bar represents the geometric mean titer $4-6$ mice + s.d. ${ }^{*} P<0.05$ by Mann-Whitney $U$. Horizontal line indicates the limit of detection of the assay.

important in protecting the intestine from infection ${ }^{25}$ whereas others support a possible role for IgA. ${ }^{16,25-27}$ The latter studies do not prove that IgA alone is critical to immunity because there was a deficiency in both IgM and IgA. ${ }^{25,26}$ Therefore, these along with numerous in vitro and passive immunization studies, correlations between IgA induction and pathogen clearance, as well as the preeminence of $\operatorname{IgA}$ in the intestine provide supportive, but not conclusive, proof that IgA is critical to protect mucosal surfaces from pathogens. Indeed, previous critical proof of concept studies in IgA-deficient animals failed to support this view. ${ }^{6-8,28}$ This paradox might be explained by several non-mutually exclusive caveats, including (i) the presence of redundant or compensatory immune effectors, such as $\operatorname{IgM}, 28$ (ii) IgA being a component of but not truly critical to immunity or to the pathogens studied, or (iii) IgA having a different or more subtle role in protective immunity than was assessed. By contrast, we show that intestinal IgA is functionally important to timely clearance of a primary infection and is absolutely essential to and can provide complete protection of the intestine from rotavirus re-infection.

Our current finding that IgA is critical for intestinal immunity to rotavirus are consistent with findings in $\mathrm{JhD}, \mu \mathrm{MT}$, $\mathrm{J}_{\text {-chain }}{ }^{-/-}$mice, all of which lack intestinal secretory antibody (IgA and IgM) and the backpack transfer of IgA that protected against rotavirus infection. ${ }^{13-18}$ Taken together, all of these previous studies suggested but did not prove the necessity of IgA to rotavirus immunity and were inconsistent with our previous finding in C57BL/6/129 IgA ${ }^{-1-}$ mice. ${ }^{11}$ One difference between the studies is the genetic background of the IgA ${ }^{-1-}$ mice. Based on the single nucleotide polymorphism (SNP) analysis, the $\mathrm{C} 57 \mathrm{BL} / 6$ and $\mathrm{BALB} / \mathrm{c} \mathrm{IgA}{ }^{-1-}$ are $>99 \% \mathrm{~B} 6$ or $>98 \% \mathrm{BALB} / \mathrm{c}$, respectively except for the Igh gene locus on chromosome 12 . By contrast, the C57BL/6/129 IgA ${ }^{-1-}$ mice used previously exhibited an approximately even distribution of 129-related and C57BL/6-related genes. It is possible that such a large representation of 129 genes may have influenced the results of our previous examination of rotavirus infection in the $\mathrm{IgA}^{-/-}$mice. Alternatively, it is possible that other confounding factors, such as the intestinal microbiota ${ }^{29}$ or concurrent infections with Helicobacter and murine norovirus, all of which can indirectly influence intestinal immunity, ${ }^{30}$ inadvertently affected the rotavirus immune response leading to the erroneous conclusion that IgA was not required for rotavirus immunity. ${ }^{11}$ In light of our new conclusions as to the importance of IgA in rotavirus immunity, perhaps a re-examination of the importance of $\operatorname{IgA}$ in the context of a more controlled genetic background is necessary for other mucosal pathogens, including Helicobacter, Herpes simplex, and influenza. ${ }^{6-8}$

Because rotavirus is commonly present in the systemic circulation during intestinal infection, ${ }^{31}$ we examined whether the loss of IgA impacted rotavirus systemic infection, clearance or protection. The levels of antigen in the blood were not elevated in the absence of $\operatorname{IgA}$, which was consistent with the induction of wild-type levels of serum antibody in the $\operatorname{Ig} \mathrm{A}^{-1-}$ mice. Unlike the importance of $\operatorname{IgA}$ in rotavirus clearance and protective immunity in the intestine, IgA has only a minimal role in clearance of the virus from the blood and is not essential for protective immunity against systemic virus upon re-exposure to the virus (Figure 5). However, B cells are important for the clearance of virus from the systemic circulation. ${ }^{15}$ Together, these studies implicate either systemic IgG or IgM as the important B-cell effector product in removal of systemic virus. This is not an unexpected conclusion as rotavirus infection induces 
substantially higher levels of serum IgG than IgA. ${ }^{22}$ In addition, $\operatorname{IgG}$ rather than IgA, is the most abundant antibody isotype in the serum, ${ }^{1}$ and thus probably has a key role in clearance of rotavirus in the blood and in protection against antigenemia and viremia upon re-exposure.

The loss of IgA results in a wide range of clinical responses to pathogenic infections in humans, ${ }^{4}$ but to our knowledge, the impact on rotavirus infections has not been examined. Based on our mouse studies, rotavirus infections in IgA-deficient humans would be expected to be more prolonged or occur more frequently compared with IgA normal individuals. Disease severity might also be increased. Importantly, rotavirus vaccines might be less efficacious in humans with IgA-deficiency syndromes, although the immune mechanism of protection is not known for either the RotaRix ${ }^{\oplus}$ or RotaTeq ${ }^{\circledR}$ vaccines. Alternatively, compensatory increases in rotavirus-specific secretory IgM in the IgA-deficient human intestine might provide greater levels of protection than we observed in the mouse studies. ${ }^{28}$ Studies in children indicate that stool IgA correlates with susceptibility to rotavirus infection, as children whose IgA levels declined following an initial rotavirus infection were not protected from re-infection. ${ }^{32}$ Clearly, the importance of IgA to rotavirus immunity found in mice re-emphasizes the importance of considering stool IgA as a correlate of rotavirus protection in human studies.

We definitively demonstrate that IgA is important for timely clearance of rotavirus infection and is absolutely essential in protection from re-infection. Although IgA is the most abundant antibody isotype in the GI tract and is synthesized in quantities that far exceed the other isotypes, ${ }^{1}$ multiple studies in both animals and humans lacking or deficient in only IgA generally failed to prove that IgA was critical for protective immunity. ${ }^{4}$ We provide new evidence that proves the importance of IgA by demonstrating that the protective immune response to a common intestinal viral enteropathogen absolutely depends on the presence of IgA and lends needed credence to the important role of IgA in pathogen-specific intestinal immunity. Our findings reinforce the need to pursue intestinal IgA as an area for exploitation in the development of highly efficacious mucosal vaccines to prevent GI infections. GI infections, including rotavirus, account for direct costs of over $\$ 7.3$ billion dollars to the US healthcare industry, and non-food-and food-borne gastroenteritis are the most common reasons for emergency room visits. ${ }^{33}$

\section{METHODS}

IgA knockout mice. C57BL/6/129 IgA ${ }^{-/-}$mice were initially generated as described previously. ${ }^{5} \mathrm{C} 57 \mathrm{BL} / 6 / 129$ mice were backcrossed $>8$ generations to either the $\mathrm{C} 57 \mathrm{BL} / 6$ or BALB/c backgrounds. Tail DNA from each resulting knockout strain was assessed at the DartMouse Speed Congenic Core Facility at Dartmouth Medical School (Hannover, NH). DartMouse uses the Illumina, (San Diego, CA) GoldenGate Genotyping Assay to interrogate 1,449 SNPs spread throughout the genome. The raw SNP data were analyzed using DartMouse's proprietary SNaP-Map and Map-Synth software, allowing the determination for each mouse of the genetic background at each SNP location. SNP analysis confirmed the backcrossing results and showed that although the Igh locus on chromosome 12 remained 129, there were few, if any,
129 gene segments detectable in other loci $(<1 \%$ C57BL6 or $<2 \%$ $\mathrm{BALB} / \mathrm{c}$ ). Both female and male mice of at least 6 weeks of age were used in experiments. Each experiment was performed a minimum of three separate times with five mice in each group. Mice were individually housed in microisolator cages, fed ad libitum, and a 24 -h random fecal sample collected every other day. Procedures were carried out within the provisions of the Guide for the Care and Use of Laboratory Animals, NIH Guide for Grants and Contracts and approval for the study was obtained from the Institutional Animal Care and Use Committee at Baylor College of Medicine. All animals were cared for under veterinary surveillance.

Viral infection, sample collection, and processing. The murine strain of rotavirus, $\mathrm{EC}_{\mathrm{wt}}$, was obtained from Harry Greenberg (Stanford University Medical School, Palo Alto, CA). Intestinal lysates were prepared from suckling mice inoculated orally with $\mathrm{EC}_{\mathrm{wt}}$ and the $\mathrm{ID}_{50}$ titer of the virus in the lysate was determined in adult $\mathrm{C} 57 \mathrm{BL} / 6$ and $\mathrm{BALB} / \mathrm{c}$ mice as described previously. ${ }^{24}$ Wild-type and $\mathrm{C} 57 \mathrm{BL} / 6$ or $\mathrm{BALB} / \mathrm{c} \mathrm{IgA}{ }^{-1-}$ mice were orally inoculated with $10^{3} \mathrm{ID}_{50}$ in $100 \mu \mathrm{l}$ of phosphate-buffered saline on day 0 and days 42 or 84 or on all 3 days $(0,48$, and 84$)$. Following both initial and subsequent viral exposures, 24-h randomized fecal pellets from individual mice were collected every other day starting on the day of inoculation. Pellets were resuspended as a $10 \%$ weight/volume solution in $10 \mathrm{~mm}$ Tris, $100 \mathrm{~mm} \mathrm{NaCl}, 1 \mathrm{~mm} \mathrm{CaCl} 2,0.05 \%$ Tween 20 , $5 \mathrm{~mm}$ sodium azide, $1 \mathrm{mg}$ of aprotinin/ml, $1 \mathrm{~mm}$ benzamidine, $10 \mathrm{mg}$ of leupeptin/ml, $10 \mathrm{mg}$ of pepstatin $\mathrm{A} / \mathrm{ml}(\mathrm{pH} 7.4)$ ) and then were centrifuged at $15,000 \mathrm{~g}$ to remove fecal solids. Processed fecal antibody samples were stored at $-20^{\circ} \mathrm{C}$. Serum was collected by either tail bleeding or from the inferior vena cava.

ELISA to measure rotavirus. The amount of rotavirus in either $10 \%$ weight/volume fecal pellet suspensions or undiluted serum following pretreatment with $0.05 \mathrm{M}$ EDTA was measured by ELISA as previously described. ${ }^{24}$ Briefly, 96-well polyvinylchloride plates were coated with $50 \mu \mathrm{l}$ of a mouse monoclonal anti-VP6 antibody (6E7, raised against rotavirus strain SA11) and incubated overnight at room temperature. Hyperimmune guinea pig anti-serum to rotavirus (strain SA11) was used to detect antigen in samples followed by horseradish peroxidase-conjugated goat anti-guinea pig IgG (Sigma-Aldrich, St Louis, MO). Values with an optical density (OD) @ $450 \mathrm{~nm}>0.100$ were considered positive. To allow for quantitative comparison of the amount of rotavirus antigen excreted in stool at the peak of infection when ELISA OD readings were saturated (>1.00), stools were serially diluted twofold, endpoint titers determined for individual samples, and geometric mean titers calculated for each group. The antigen titer was defined as the reciprocal of the highest dilution resulting in an $\mathrm{OD}>0.100$.

ELISA to measure anti-rotavirus $\lg A, \lg G$, and $\lg M$ in feces and serum. The ELISA to measure rotavirus-specific IgA, IgG, and IgM has been previously described. ${ }^{24}$ Briefly, plates were coated with an anti-SA11 hyperimmune guinea pig serum followed by incubation with a lysate of SA11-infected MA104 cells treated with 0.05 M EDTA. Incubation of SA11 lysate was followed by incubation of either fecal suspensions or serum with an initial dilution of $1 / 2$ or $1 / 50$, respectively. The amount of antibody was quantified following incubation with a horseradish peroxidase-conjugated goat anti-mouse IgA, IgG, and IgM (Kirkegaard and Perry Laboratories Inc., Gaithersburg, MD) diluted in buffer containing normal guinea pig serum. Values with an $\mathrm{OD}>0.100$ were considered positive. The antibody titer was defined as the reciprocal of the highest dilution resulting in an $\mathrm{OD}>0.100$.

Statistical analysis. $\chi^{2}$ testing (Fisher's Exact) followed by KruskalWallis equality-of-populations rank test was used to determine statistical significance between groups ( $n=5-12$ mice/group). Antibody titers between groups were compared by the Kruskal-Wallis test to determine statistical significance followed by a one-sided Mann-Whitney 
$U$ test to determine the $P$ values of groups that had statistically significant differences. Statistical significance was concluded when $P<0.05$.

\section{ACKNOWLEDGMENTS}

This work was supported, in part, by Public Health Service Grants NIH Al24998 (S.E.B, A.M, and M.E.C), NIH Al07471 (A.M.) and NIH Al41715 and $\mathrm{NIH}$ Al83878 (S.L.S, and D.W.M.), as well as NIH DK56338, which funds the Texas Medical Center Digestive Diseases Center. Technical assistance was provided by Samuel Hardeman, Lara Berghammer, and Mary Penn.

\section{DISCLOSURE}

The authors declare no conflict of interest.

() 2012 Society for Mucosal Immunology

\section{REFERENCES}

1. Janeway, C., Travers, P., Walport, M. \& Shlomckik, J. Immunobiology: The Immune System in Health and Disease (Elsevier Science/Garland Publishing, London, 2001).

2. Mestecky, J., Moro, I. \& Underdown, J. Mucosal immunoglobins. In Mucosal Immunology (Ogra, P., et al., eds) 133-152 (Academic Press, San Diego, 1999).

3. Cerutti, A., Chen, K. \& Chorny, A. Immunoglobulin responses at the mucosal interface. Annu. Rev. Immunol. 29, 273-293 (2011).

4. Yel, L. Selective IgA deficiency. J. Clin. Immunol. 30, 10-16 (2010).

5. Harriman, G. R. et al. Targeted deletion of the IgA constant region in mice leads to IgA deficiency with alterations in expression of other ig isotypes. J. Immunol 162, 2521-2529 (1999).

6. Blanchard, T.G. et al. Antibody-independent protective mucosal immunity to gastric Helicobacter infection in mice. Cell Immunol. 191, 74-80 (1999)

7. Parr, M.B., Harriman, G.R. \& Parr, E.L. Immunity to vaginal HSV-2 infection in immunoglobulin A knockout mice. Immunology 95, 208-213 (1998).

8. Mbawuike, I.N. et al. Mucosal immunity to influenza without IgA: an IgA knockout mouse model. J. Immunol. 162, 2530-2537 (1999).

9. Silvey, K.J., Hutchings, A.B., Vajdy, M., Petzke, M.M. \& Neutra, M.R. Role of immunoglobulin A in protection against reovirus entry into Murine Peyer's patches. J. Virol. 75, 10870-10879 (2001).

10. Estes, M.K. \& Kapikian, A.Z. Field's virology. In (Knipe, D.M. \& Howley, P. M. eds) 1917-1974 (Lippincott-Raven Publishers, Philadelphia, 2007).

11. O'Neal, C.M., Harriman, G.R. \& Conner, M.E. Protection of the villus epithelial cells of the small intestine from rotavirus infection does not require immunoglobin A. J. Virol. 74, 4102-4109 (2000).

12. Kuklin, N.A. et al. Protective intestinal anti-rotavirus $B$ cell immunity is dependent on alpha 4 beta 7 integrin expression but does not require IgA antibody production. J. Immunol. 166, 1894-1902 (2001).

13. Franco, M.A. \& Greenberg, H.B. Role of B cells and cytotoxic T lymphocytes in clearance of and immunity to rotavirus infection in mice. J. Virol. 69, 7800-7806 (1995).

14. McNeal, M.M., Barone, K.S., Rae, M.N. \& Ward, R.L. Effector functions of antibody and CD8+ cells in resolution of rotavirus infection and protection against reinfection in mice. Virology 214, 387-397 (1995).
15. Marcelin, G., Miller, A.D., Blutt, S.E. \& Conner, M.E. Immune mediators of rotavirus antigenemia clearance in mice. J. Virol. 85, 7937-7941 (2011).

16. Burns, J.W., Siadat-Pajouh, M., Krishnaney, A.A. \& Greenberg, H.B. Protective effect of rotavirus VP6-specific IgA monoclonal antibodies that lack neutralizing activity. Science 272, 104 (1996).

17. McNeal, M.M. et al. Protection against rotavirus shedding after intranasal immunization of mice with a chimeric VP6 protein does not require intestinal lgA. Virology 346, 338-347 (2006).

18. Schwartz-Cornil, I., Benureau, Y., Greenberg, H., Hendrickson, B.A. \& Cohen, J. Heterologous protection induced by the inner capsid proteins of rotavirus requires transcytosis of mucosal immunoglobulins. J. Virol. 76, 8110-8117 (2002).

19. Brandtzaeg, P. \& Prydz, H. Direct evidence for an integrated function of $J$ chain and secretory component in epithelial transport of immunoglobulins. Nature 311, 71-73 (1984).

20. Blutt, S.E., Warfield, K.L., O’Neal, C.M., Estes, M.K. \& Conner, M.E. Host, viral, and vaccine factors that determine protective efficacy induced by rotavirus and virus-like particles (VLPS). Vaccine 24, 1170-1179 (2006).

21. Sanford, L.P., Kallapur, S., Ormsby, I. \& Doetschman, T. Influence of genetic background on knockout mouse phenotypes. Methods Mol. Biol. 158, 217-225 (2001).

22. Franco, M.A. \& Greenberg, H.B. Immunity to homologous rotavirus infection in adult mice. Trends Microbiol. 8, 50-52 (2000).

23. Blutt, S.E. \& Conner, M.E. Rotavirus: to the gut and beyond!. Curr. Opin. Gastroenterol. 23, 39-43 (2007).

24. O'Neal, C.M., Crawford, S.E., Estes, M.K. \& Conner, M.E. Rotavirus VLPs administered mucosally induce protective immunity. J. Virol. 71, 8707-8717 (1997).

25. Uren, T.K. et al. Vaccine-induced protection against gastrointestinal bacterial infections in the absence of secretory antibodies. Eur. J. Immunol. 35, 180-188 (2005).

26. Wijburg, O.L. et al. Innate secretory antibodies protect against natural Salmonella typhimurium infection. J. Exp. Med. 203, 21-26 (2006).

27. Silvey, K.J., Hutchings, A.B., Vajdy, M., Petzke, M.M. \& Neutra, M.R. Role of immunoglobulin A in protection against reovirus entry into Murine Peyer's patches. J. Virol. 75, 10870-10879 (2001).

28. Brandtzaeg, P. et al. The clinical condition of IgA-deficient patients is related to the proportion of IgD- and lgM-producing cells in their nasal mucosa. Clin. Exp. Immunol. 67, 626-636 (1987).

29. Reading, N.C. \& Kasper, D.L. The starting lineup: key microbial players in intestinal immunity and homeostasis. Front. Microbiol. 2, 148 (2011).

30. Bernasconi, N.L., Traggiai, E. \& Lanzavecchia, A. Maintenance of serological memory by polyclonal activation of human memory B cells. Science 298, 2199-2202 (2002).

31. Blutt, S.E. et al. Rotavirus antigenaemia and viraemia: a common event? Lancet 362, 1445-1449 (2003).

32. Coulson, B.S. et al. Comparison of rotavirus immunoglobulin $A$ coproconversion with other indices of rotavirus infection in a longitudinal study in childhood. J. Clin. Microbiol. 28, 1367-1374 (1990).

33. Sandler, R.S. et al. The burden of selected digestive diseases in the United States. Gastroenterology 122, 1500-1511 (2002). 